\title{
Tadalafil Rehabilitation Therapy Preserves Penile Size After Bilateral Nerve Sparing Radical Retropubic Prostatectomy
}

\author{
Ozgu Aydogdu, Mehmet Ilker Gokce, Berk Burgu, Sumer Baltacı, Onder Yaman \\ Ankara University School of Medicine, Department of Urology, Ankara, Turkey
}

\begin{abstract}
Objective: To evaluate the change in penile size $r$ bilateral nerve sparing retropubic radical prostatectomy (BNSRRP) and possible effect of Tadalafil.

Materials and Methods: A total of 65 patients underwent BNSRRP and they were evaluated prospectively for a whole year of follow-up. The patients were randomized to control without rehabilitation (Group 1) or Tadalafil rehabilitation group (Group 2). The patients were evaluated at months 3, 6 and 12 postoperatively for erectile function, penile measurements (flaccid penile length, penile length at maximum erection, penile circumference at flaccid status, and penile circumference at maximum erection), penile abnormalities and general health status. Statistical analysis was performed by Chi-Square test and significance was defined as $\mathrm{p}$ value $<0.05$.

Results: In Group 1 there was significant decrease in penile measurements at month 3 compared to preoperative measurements. There was decrease in all parameters at month 6 compared to month 3 but only the decrease in penile length at maximum erection was significant. There were no significant differences between postoperative months 6 and 12 for all measurements. In Group 2 there was a tendency to decrease in all measurements at month 3 compared to baseline. There was no significant difference for penile measurements between postoperative 3rd and 6th months and between 6th month and the first year.

Conclusion: Although further large sampled trials are needed to describe the possible positive effect of tadalafil or other PDE5-I's on penile size after BNSRRP, tadalafil rehabilitation is effective in preserving penile size especially in the early postoperative period after BNSRRP.
\end{abstract}

Key words: Penile: erectile; prostatectomy; erectile dysfunction; tadalafil

Int Braz J Urol. 2011; 37: 336-346

\section{INTRODUCTION}

In recent years especially with the advent of serum prostate specific antigen (PSA) prostate cancer has been frequently detected in relatively young men (1). Besides other treatment modalities radical prostatectomy (RP) is generally used for patients with clinically localized prostate cancer (2). The goal of the surgical intervention is to eradicate the disease with minimal possible morbidity. Erectile dysfunction (ED) and urinary incontinence are the two main potential problems that may adversely affect the patients' quality of life postoperatively (3).

In different series ED was reported in 14$84 \%$ of the men who underwent RP (4-6). Men suffering from postoperative ED may also complain from loss of penile length (7). Additionally some patients without postoperative ED may not have desirable sexual intercourse because of penile shortening (8). Some studies in the literature prospectively tested the use of phosphodiesterase 5 inhibitors (PDE5-I) and their potential effect on postoperative erectile function after RP $(9,10)$. 
Few trials studied the possible effect of PDE5-I's specifically on penile length in the postoperative period (8).

In this prospective study we aimed to evaluate the change in penile size after bilateral nerve sparing retropubic radical prostatectomy (BNSR$\mathrm{RP}$ ) and possible effect of tadalafil treatment in a relatively young population with prostate cancer. We hypothesized that presence of postoperative ED and the protocol of postoperative tadalafil administration as rehabilitation therapy might affect the penile length.

\section{MATERIALS AND METHODS}

Between October 2006 and February 2008, 85 patients with full preoperative potency underwent BNSRRP in our institution. Data of 65 of these patients were evaluated in this prospective trial and flow chart for the patient enrollment is summarized in Figure-1. Inclusion criteria were as follows: age $<65$ years, preoperative full potency, no history of penile plaques or previous penile surgery, clinical stage T1c or lower, PSA $<10 \mathrm{ng} / \mathrm{mL}$ and a biopsy Gleason score $<8$. All patients were preoperatively evaluated by International Index of Erectile Function (IIEF-EF) and Sexual Encounter
Profile (SEP) diary questions 2 and 3 to determine the erectile function status of the patient. All patients had preoperative IIEF-EF scores $>25$ and answered SEP questions 2-3 'yes'. General health status was questioned on co-morbidities involving hyperlipidemia, diabetes and hypertension. Also all patients were evaluated for metabolic syndrome based on the guidelines from the 2001 National Cholesterol Education Program Adult Treatment Panel (ATP III) (11). Physical examination and penile ultrasound scan were performed preoperatively to detect any penile plaque or abnormality. Penile length and circumference were measured both in flaccid and at maximum erection with a paper ruler as reported by Munding et al. (12), by a single physician. Penile length was measured from the tip of the glans to the pubopenile skin junction; penile circumference was measured at the most distal part of the shaft, just proximal to the glans penis. Measurements were repeated twice to optimize the reliability of the results obtained. To obtain maximum erection, $30 \mathrm{mg}$ of papaverine was injected to each corpus cavernosa and patients were required to do manual genital stimulation.

All patients underwent BNSRRP by the technique previously described by Montorsi et al. (6) that is famous for permitting efficient dissec-

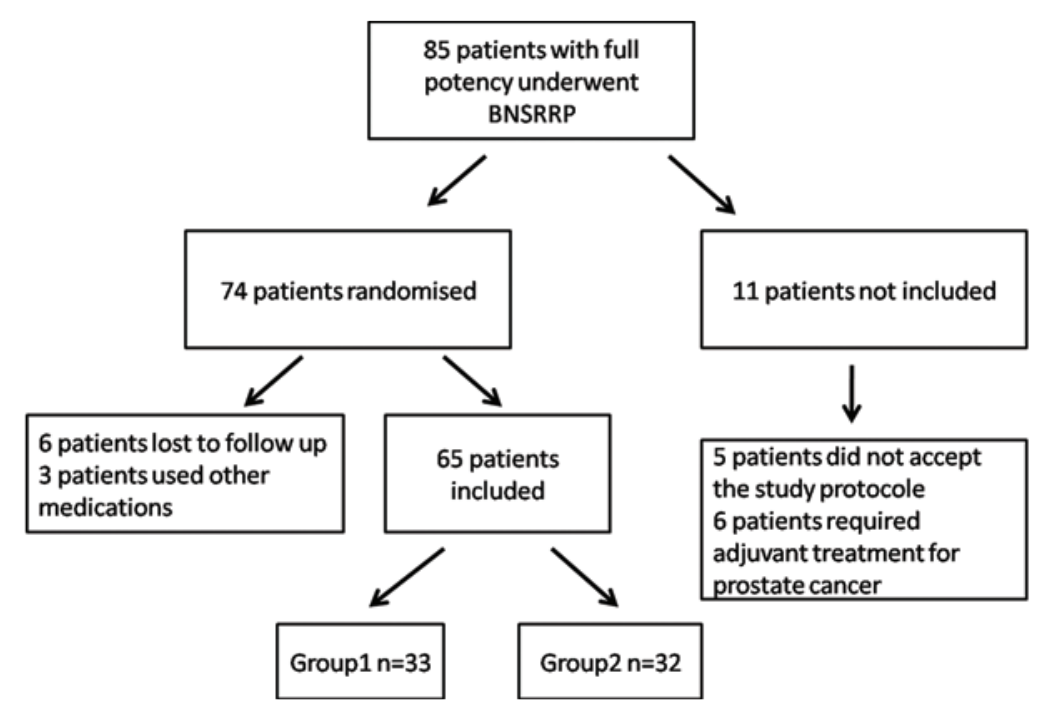

Figure 1 - Flow chart for the inclusion of the patients. 
tion of the neurovascular bundles at the level of the membranous urethra and prostate apex, which significantly reduces the risk of cavernous nerve injury. The urethral catheter was removed 14-20 days after the surgery and the patients were encouraged to attempt sexual intercourse 1 week after the catheter removal. The patients were randomized to two groups; no use of tadalafil (Group 1) and 3 days/week tadalafil (20mg/day) protocols (Group 2) on the day of surgery. For randomization NCSS (Number Cruncher Statistical System) 2007 program was used. Patients in Group 1 and Group 2 received the protocols for 6 months following the removal of urethral catheter.

The patients were evaluated at months 3,6 and 12 following the surgery for erectile function, penile measurements, penile abnormalities and general health status with the same protocol used preoperatively. Penile size measurements were performed blindly by the same physician preoperatively and postoperatively. None of the patients received any adjuvant or neoadjuvant treatment for prostate cancer.

Statistical evaluations were performed with Chi-Square test. Two-sided tests with significance at 0.05 were used. An independent sample $t$ test was used to evaluate differences in penile measurements and erectile function between the pre and postoperative periods. All patients signed a written informed consent before the randomization in the study.

\section{RESULTS}

Patients mean age was 57.2 years old (50 to 65 years old). Mean preoperative PSA was 6 , $1 \mathrm{ng} / \mathrm{mL}$ (3.6-9.6ng/mL). Prostate biopsy Gleason score was $<6$ in 14 patients, 6 in 56 patients and 7 in the remaining 35 patients. The three groups did not show statistical differences in preoperative factors regarding to age, PSA, IIEF-EF, hypertension, Diabetes Mellitus, hyperlipidemia and metabolic syndromes, and the values are summarized in Table-1.

The two groups did not show significant difference for the preoperative measurements. The average of pre-operative and postoperative (months 3, 6 and 12) penile measurements for both groups are shown in Figure-2. Individual penile measurements average changes for the same time periods is demonstrated in Table-2.

In Group 1 there was significant decrease in penile length and circumference measurements in both flaccid state and at maximum erection at month 3 compared to preoperative measurements $(\mathrm{p}<0.05)$. Also there was a decrease in all parameters at month 6 compared to month 3 but the only statistically significant decrease was in penile length at maximum erection $(\mathrm{p}<0.05)$. No statistical differences were noted between postoperative month 6 and 12 for all measurements ( $>$ $0.05)$.

In Group 2 there was a tendency to decrease in all measurements at month 3 compared to baseline but this decrease was not statistically significant. Also there was no statistically significant difference for any of the penile measurements between postoperative $3 \mathrm{rd}$ and 6 th months as well as between postoperative 6th month and the first year.

The comparison of the means of individual differences for penile measurements in both groups, which were performed to omit the effect of some outliers, revealed similar results with the comparison of means of each group.

Concerning erectile function status, 24 patients $(72.7 \%)$ in Group 1 and 25 in Group 2 (78.1\%) were found to have IIEF-EF $>25$ at the end of 1 year follow-up. In patients with a postoperative IIEF-EF score $>25$, the mean of individual changes of penile measurements at both flaccid status and maximum erection, between the preoperative and postoperative first year, were compared for both groups. There was no significant difference in these parameters. Although there was a prominent tendency of decrease in penile measurements for patients in both groups with a postoperative IIEF-EF score $<25$, statistical significance could not be tested due to the low number of patients. The number of patients for each group in each time period for erectile function status is given in Table-3. During the follow-up 2 patients in Group 1 developed penile plaque that were also detected by ultrasound scan. 
Table 1 - Preoperative data of both groups.

\begin{tabular}{lccc}
\hline Parameter & Group 1 (n= 33) & Group 2 (n= 32) & P value \\
\hline Age (year) & 58.1 & 56.2 & \\
PSA (ng/mL) & 5.8 & 6.3 & \\
IIEF & 26.5 & 26.2 & \\
Hypertension (n) & 12 & 14 & P $>0.05$ \\
Hyperlipidemia (n) & 6 & 4 & \\
Diabetes mellitus (n) & 4 & 5 & \\
Metabolic syndrome (n) & 6 & 5 & \\
\hline
\end{tabular}

\section{DISCUSSION}

Potential pathophysiology of ED that may occur after RP has been widely discussed in several studies (13-15) and there are studies evaluating the penile length changes after RP $(7,12,16)$. The mechanism responsible for the reduction in penile length after RP has not been elucidated. Ciancio and Kim reported that $41 \%$ of patients with ED after RP had penile fibrotic changes (17). McCullough and Lepor speculated that the loss of penile size in impotent patients after nerve sparing RRP was due to postoperative denervation atrophy and/or fibrosis of the corpora cavernosa smooth muscle (18). Iacono et al. evaluated histomorphological alterations in cavernous smooth muscle and collagen content after radical prostatectomy. Similarly to other studies, they concluded that progressive fibrosis in the corpora cavernosa after radical prostatectomy resulted from denervation and/or an ischemic process (19). Several human and animal studies support the hypothesis of maintaining adequate penile oxygenation by preserving neurovascular bundles and thus erectile function, may play an important role in preventing irreversible penile fibrotic changes $(8,14,15)$. User et al. performed a study on postpubertal rats and grouped them according to bilateral or unilateral cavernous nerve transection versus a sham operation (14). They suggested the hypothesis that bilateral injury to the cavernous nerves may induce significant apoptosis of smooth muscle cells particularly in the subtunical area which causes penile fibrosis and abnormality of the veno-occlusive mechanism of the corpus cavernosum. Leungwattanakij et al. showed that cavernosal neurotomy was associated with an up regulation of fibrogenic cytokines and collagenization of the corporoa smooth muscle in animal model (15). In many studies it has been postulated that a chronic absence of postoperative erections might lead to cavernosal hypoxia $(7,12,16)$. Finally, significant tissue damage and increased corpora fibrosis occur and may cause postoperative shortening of the penis.

Schwarts et al. found an association between early use of high doses of sildenafil after RP and preservation of smooth muscle content within human corpus cavernosum (20). Bianco et al. speculated that the surgeon plays the most important role in determining the patients' postoperative outcome (21).

In our trial, operations were performed by two high volume surgeons. The mean preoperative flaccid penile dimensions in our series were similar to those reported before $(16,22)$. Although the mechanisms were not well understood it's clear that a decrease in penile length may occur after RP. Our outcomes for both groups were similar to past trials suggesting a decrease in penile length postoperatively $(7,12,16)$. Briganti et al. found no difference in penile size between pre and postoperative measurements with the use of full dose PDE5-I on demand (8). We found a significant decrease in all penile measurements in control patients (not using Tadalafil) at 3 rd postoperative month when compared with preoperative measurements $(p=0.001)$. There was also a significant decrease in penile length measured at maxi- 
Figure 2 - The means and standard deviations (SD) of penile measurements preoperatively and at months 3, 6 and 12 postoperatively for both groups.
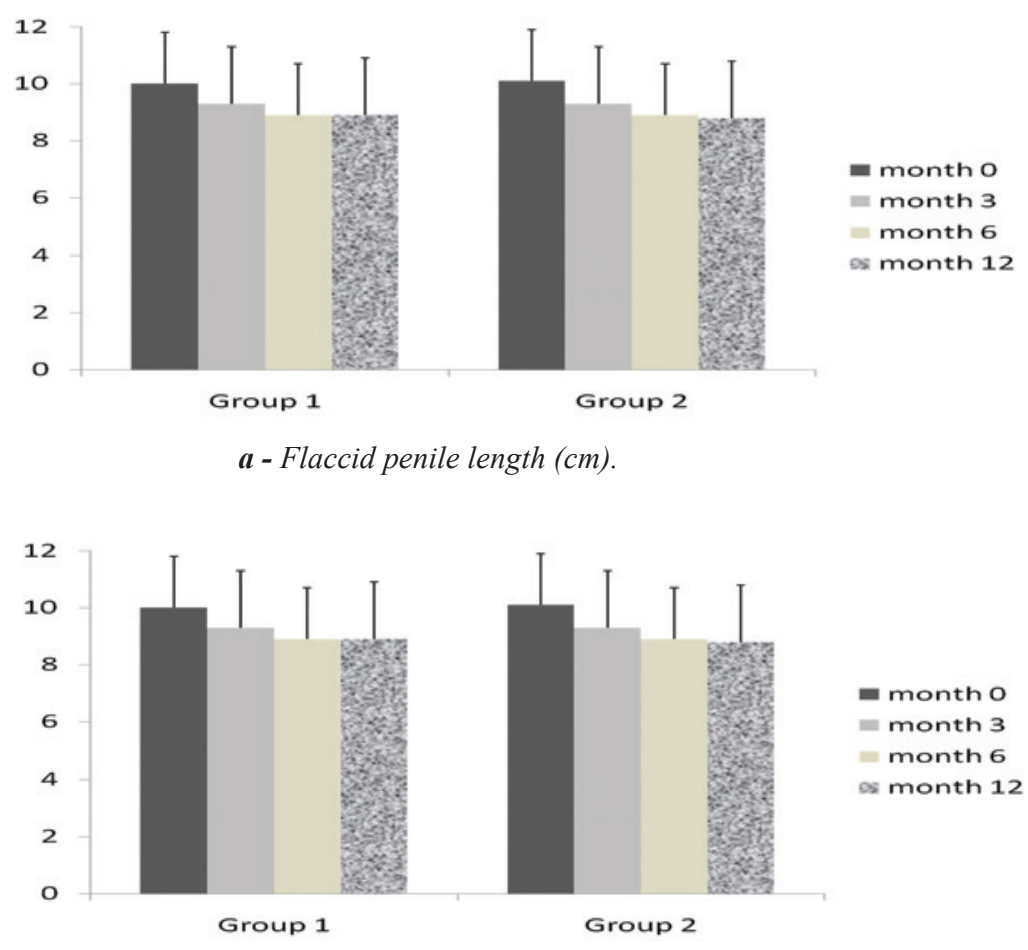

$\boldsymbol{b}$ - Penile length at maximum erection (cm).

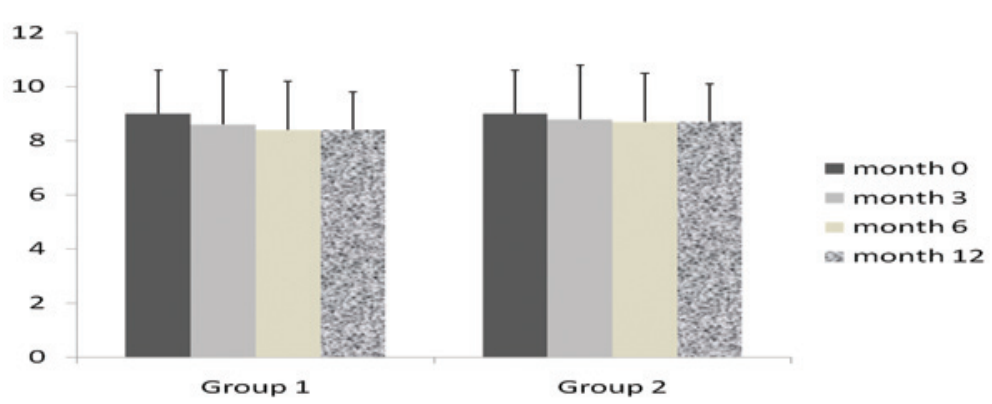

c - Penile circumference at flaccid status $(\mathrm{cm})$.

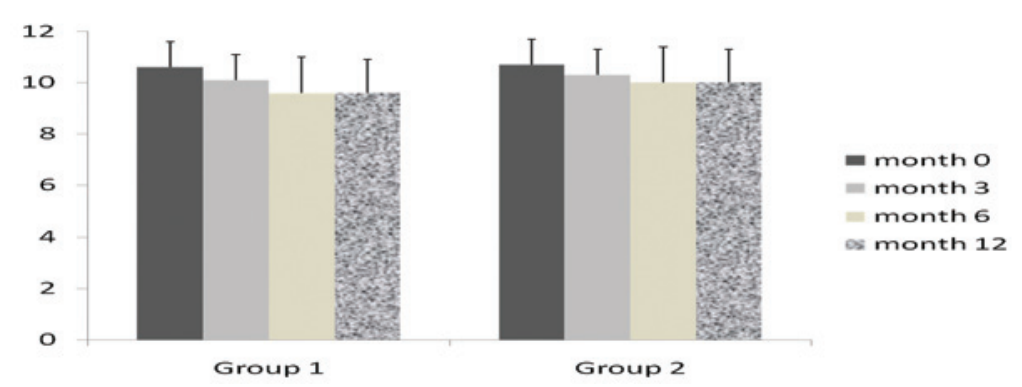

$\boldsymbol{d}$ - Penile circumference at maximum erection (cm). 
Table 2 - Follow-up of changes of penile measurements (mean) of groups 1 and 2

\begin{tabular}{lcc}
\hline & Group 1 (Mean) & Group 2 (Mean) \\
\hline Change in flaccid penile length (cm) & $0.8^{*}$ & 0.5 \\
0-3 Months & 0.3 & 0.2 \\
3-6 Months & 0.1 & 0.1 \\
6-12 Months & & 0.6 \\
Change in penile length at maximum erection (cm) & $0.9^{*}$ & 0.4 \\
0-3 Months & $0.7^{*}$ & 0.1 \\
3-6 Months & 0.2 & 0.1 \\
6-12 Months & & 0.3 \\
0-3 Months & $0.5^{*}$ & 0.1 \\
3-6 Months & 0.2 & 0.1
\end{tabular}

Change in penile circumference at maximum erection

(cm)

$\begin{array}{lcr}\text { 0-3 Months } & 0.4^{*} & 0.3 \\ \text { 3-6 Months } & 0.1 & 0.1 \\ \text { 6-12 Months } & 0.1 & 0.1\end{array}$

* mean $p<0.05$

mum erection at 6 months compared to 3 months postop for the first group. In patients who used Tadalafil regularly as a rehabilitation protocol in Group 2, there was no statistically significant penile shortening at 3 months postop when compared to preoperative measures $(\mathrm{p}=0.07)$. Regular use of Tadalafil revealed similar results as Briganti's stduy (8). Despite the limited number of patients in our series, we believe that early use of Tadalafil as a rehabilitation therapy may have positive effects on penile length after RP.

Although Savoie et al. (16) reported penile shortening in $68 \%$ of their patients following RP; they concluded that there was no difference between patients with or without postoperative ED in terms of penile measurements. However Briganti et al. showed that postoperative preservation of erectile function is positively correlated with the maintenance of penile length following BNSRRP (8). Likewise to their results our patients who had an IIEF-EF score $>25$, had preservation of penile size following surgery. However, we could not verify meaningful results in patients with IEFEF score $<25$ postoperatively, probably due to the low number of patients. Therefore, we can not conclude an association between preservation of postoperative erectile function and penile length.

There is a lack of consensus on penile measurements and the use of PDE5-I's following RP and presence (unilateral or bilateral) or absence of nerve sparing surgery and the type of protocol of PDE5-I (on demand/or no use vs. rehabilitation). Although we did not evaluate the effect of nerve sparing surgery on penile measurements, we compared pre and postoperative penile measurements in both 'penile rehabilitation' and 'control' groups.

Sildenafil has been previously shown to be associated with maintenance of postoperative penile structures in patients undergoing RP (20). However it was reported in a recent study that 
Table 3 - Erectile function status of patients in both groups.

\begin{tabular}{|c|c|c|c|c|}
\hline \multicolumn{5}{|c|}{ Group1 $(n=33)$} \\
\hline IIIEF & $\begin{array}{l}\text { Month 0 } \\
\text { (bas eline) }\end{array}$ & Month 3 & Month 6 & Month 12 \\
\hline $6-10$ & $-\bullet$ & - & 1 & $1 \bullet$ \\
\hline $11-16$ & $-\bullet$ & 5 & 5 & $4 \bullet$ \\
\hline $17-25$ & $-\bullet$ & 9 & 8 & $9 \bullet$ \\
\hline $26-30$ & $33 \dagger$ & 19 & 19 & $19 \dagger$ \\
\hline \multicolumn{5}{|l|}{ SEP 2} \\
\hline Yes & 33 & 22 & 22 & 21 \\
\hline No & - & 11 & 11 & 12 \\
\hline \multicolumn{5}{|l|}{ SEP 3} \\
\hline Yes & 33 & 23 & 22 & 22 \\
\hline No & - & 10 & 11 & 11 \\
\hline \multicolumn{5}{|c|}{ Group $2(n=32)$} \\
\hline IIEF & $\begin{array}{l}\text { Month } 0 \\
\text { (bas eline) }\end{array}$ & Month 3 & Month 6 & Month 12 \\
\hline $6-10$ & $-\bullet$ & 1 & - & 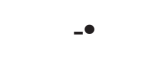 \\
\hline $11-16$ & $\bullet$ & 3 & 4 & $3 \bullet$ \\
\hline $17-25$ & $-\bullet$ & 7 & 7 & $8 \bullet$ \\
\hline $26-30$ & $32 \dagger$ & 21 & 21 & $21 \dagger$ \\
\hline \multicolumn{5}{|l|}{ SEP 2} \\
\hline Yes & 32 & 23 & 23 & 24 \\
\hline No & - & 9 & 9 & 8 \\
\hline \multicolumn{5}{|l|}{ SEP 3} \\
\hline Yes & 32 & 25 & 26 & 26 \\
\hline No & - & 7 & 6 & 6 \\
\hline
\end{tabular}

- = Although there was a prominent tendency of decrease in penile measurements for patients in both groups with a postoperative IIEF-EF score < 25, statistical significance could not be demonstrated probably due to the low number of patients.

$\dagger=$ In patients with a postoperative IIEF-EF score $>25$, there was no significant difference in the means of individual changes of penile measurements at both flaccid status and maximum erection, between the preoperative and postoperative 1st year for both groups.

the duration of action of sildenafil and vardenafil is about 4 hours, whereas Tadalafil action is approximately 36 hours, and the overall safety of the treatment is good (23). In another study by Doggrell S (24) it was concluded that Tadalafil is similarly effective as Sildenafil in the treatment of ED and in comparison studies; Tadalafil is preferred to Sildenafil $(50 / 100 \mathrm{mg})$ by men with ED, possibly because of its longer duration of action.

Our study represents a prospective study including relatively young and preoperatively potent patients who underwent BNSRRP and post- operatively underwent 'Tadalafil rehabilitation' or 'no rehabilitation-control' groups. The duration of action of Tadalafil which is 36 hours provided a PDE5 inhibition in the rehabilitation group.

Preservation of penile size in a man with ED may be crucial to be potential candidates for penile implantation. In addition, we question whether ED may be the result of decreased self-esteem due to shortened penile size following surgery.

We observed a significant decrease in all penile measurements of the control patients in the early postoperative period ( $3 \mathrm{rd} \mathrm{month}$ ) when com- 
pared to baseline. However, this decrease was not statistically significant when early and late postoperative penile measurements were compared. There was no significant decrease in both early and late postoperative measurements when compared to preoperative penile length measurements in the rehabilitation group. These findings can also be interpreted as, postoperative Tadalafil penile rehabilitation as effective therapy in the early postoperative period, decreasing the rate of penile length shortening in patients undergoing $\mathrm{BN}$ SRRP in the first postoperative year.

Despite our findings, the study is limited by a relatively small sample size and there is no evaluation regarding the effect of nerve sparing (unilateral vs. bilateral) surgical technique. Although all patients were encouraged to have sexual intercourse after the removal of the urethral catheter, not all men were motivated do to so after surgery.

\section{CONCLUSIONS}

Tadalafil penile rehabilitation is an effective therapy in preserving penile size especially in the early postoperative period after BNSRRP. Future clinical trials with larger population of patients may better define the possible positive effects of PDE5-I's on penile size after BNSRRP, including Tadalafil.

\section{CONFLICT OF INTEREST}

None declared.

\section{REFERENCES}

1. Ries L. A. G., Kosary C. L., Hankey B. F. et al. SEER Cancer Statistic Review, 1973-1994. National Institutes of Health Publication, No. 97-2789. Bethesda, Maryland: National Cancer Institute. 1997.

2. Aus G, Abbou CC, Bolla M, Heidenreich A, Schmid HP, van Poppel H, et al.: EAU guidelines on prostate cancer. Eur Urol. 2005; 48: 546-51.

3. Penson, D. F. and Litwin, M. S. Quality of life issues in patients with prostate cancer. AUA Update
Series, vol. 20. Houston, TX: AUA Office of Education 2001;18-24.

4. Karakiewicz PI, Tanguay S, Kattan MW, Elhilali MM, Aprikian AG: Erectile and urinary dysfunction after radical prostatectomy for prostate cancer in Quebec: a population-based study of 2415 men. Eur Urol. 2004; 46: 188-94.

5. Dubbelman YD, Dohle GR, Schröder FH: Sexual function before and after radical retropubic prostatectomy: A systematic review of prognostic indicators for a successful outcome. Eur Urol. 2006; 50: 711-8; discussion 718-20.

6. Montorsi F, Briganti A, Salonia A, Rigatti P, Burnett AL: Current and future strategies for preventing and managing erectile dysfunction following radical prostatectomy. Eur Urol. 2004; 45: 123-33.

7. Fraiman MC, Lepor H, McCullough AR: Changes in Penile Morphometrics in Men with Erectile Dysfunction after Nerve-Sparing Radical Retropubic Prostatectomy. Mol Urol. 1999; 3: 109-115.

8. Briganti A, Fabbri F, Salonia A, Gallina A, Chun FK, Dehò F, et al.: Preserved postoperative penile size correlates well with maintained erectile function after bilateral nerve-sparing radical retropubic prostatectomy. Eur Urol. 2007; 52: 702-7.

9. Montorsi F, Brock G, Lee J, Shapiro J, Van Poppel $H$, Graefen $M$, et al.: Effect of nightly versus ondemand vardenafil on recovery of erectile function in men following bilateral nerve-sparing radical prostatectomy. Eur Urol. 2008; 54: 924-31.

10. Teloken P, Mesquita G, Montorsi F, Mulhall J: Post-radical prostatectomy pharmacological penile rehabilitation: practice patterns among the international society for sexual medicine practitioners. J Sex Med. 2009; 6: 2032-8.

11. Expert Panel on Detection, Evaluation, and Treatment of High Blood Cholesterol in Adults: Executive Summary of The Third Report of The National Cholesterol Education Program (NCEP) Expert Panel on Detection, Evaluation, And Treatment of High Blood Cholesterol In Adults (Adult Treatment Panel III). JAMA. 2001; 285: 2486-97.

12. Munding MD, Wessells HB, Dalkin BL: Pilot study of changes in stretched penile length 3 months after radical retropubic prostatectomy. Urology. 2001; 58: 567-9.

13. Mulhall JP: Penile length changes after radical prostatectomy. BJU Int. 2005; 96: 472-4.

14. User HM, Hairston JH, Zelner DJ, McKenna KE, McVary KT: Penile weight and cell subtype specific changes in a post-radical prostatectomy model of 
erectile dysfunction. J Urol. 2003; 169: 1175-9.

15. Leungwattanakij S, Bivalacqua TJ, Usta MF, Yang DY, Hyun JS, Champion HC, et al.: Cavernous neurotomy causes hypoxia and fibrosis in rat corpus cavernosum. J Androl. 2003; 24: 239-45.

16. Savoie M, Kim SS, Soloway MS: A prospective study measuring penile length in men treated with radical prostatectomy for prostate cancer. J Urol. 2003; 169: 1462-4.

17. Ciancio SJ, Kim ED: Penile fibrotic changes after radical retropubic prostatectomy. BJU Int. 2000; 85: 101-6.

18. McCullough AR Lepor H: The loss of penile length and circumference in impotent men after nerve sparing radical prostatectomy. J Urol. 1998; (suppl 159): 98. \# Abstract 376.

19. Iacono F, Giannella R, Somma P, Manno G, Fusco F, Mirone V: Histological alterations in cavernous tissue after radical prostatectomy. J Urol. 2005; 173: 1673-6.
20. Schwartz EJ, Wong P, Graydon RJ: Sildenafil preserves intracorporeal smooth muscle after radical retropubic prostatectomy. J Urol. 2004; 171: 771-4.

21. Bianco F, Kattan M, Eastham J, Scardino PT, Mulhall JP: Surgeon and surgical volume as predictors of erectile function outcomes following radical prostatectomy. J Sex Med. 2004; 1(suppl 1): 33. \# Abstract O15.

22. Wessells H, Lue TF, McAninch JW: Complications of penile lengthening and augmentation seen at 1 referral center. J Urol. 1996; 155: 1617-20.

23. Supuran CT, Mastrolorenzo A, Barbaro G, Scozzafava A: Phosphodiesterase 5 inhibitors--drug design and differentiation based on selectivity, pharmacokinetic and efficacy profiles. Curr Pharm Des. 2006; 12: 3459-65.

24. Doggrell S: Do vardenafil and tadalafil have advantages over sildenafil in the treatment of erectile dysfunction? Int J Impot Res. 2007; 19: 281-95.

Accepted after revision:

October 25, 2010

\section{Correspondence address:}

Dr. Ozgu Aydogdu

Ankara University Department of Urology

Adnan Saygun cad. 06100 Altındağ

Ankara, Turkey

Fax: + 0093 12 311-2167

E-mail: ozgucan@yahoo.com

\section{EDITORIAL COMMENT}

The study is very interesting. From this experiment, there are many important points for achievement of proper quality of life for men who underwent retropubic radical prostatectomy and prevention of post operative ED and loss of penile length:

The role of rehabilitation therapy by tadalafil early after BNSRP and its possible effect on the penile size and erectile function, could be due to the superiority of tadalafil over sildenafil and vardenafil by its longer duration (36 hours) and its overall safety of the treatment.

The patients were encouraged to perform sexual intercourse 1 week after catheter removal. This early performance might prevent cavernosal hypoxia.

Although the study did not evaluate the effect of surgical technique on penile measurements, the surgeon's skill is a major factor. The bilateral nerve sparing radical retropubic pros- 
tatectomy and the efficient dissection of the membranous urethra and prostatic apex reduce significant risk of cavernous nerve injury which prevents penile fibrotic changes.

Dr. Olfat El-Sibai

Professor and Chairman

Deptartment of Surgery

Faculty of Medicine

Menoufia University

Shebin El-Kom, Egypt

Dr. Ali A. Shafik

Professor of Surgery

Cairo University

President of Mediterranean Society of Coloproctol-

ogy (Rome)

E-mail:shafik@ahmedshafik.com

\section{EDITORIAL COMMENT}

The microarchitecture of erectile tissue is closely related to erectile function (1-3). As previously assumed (4), the microstructural alterations inside the corpus cavernosum are likely to have an impact on its function becoming the cause, or at least a concomitant cause, of erectile deficiency. In patients who underwent radical prostatectomy, the quantitative and qualitative decrease in elastic fibers and smooth muscle fibers, along with a progressive increase in collagen fibers deposition, leads the corpus cavernosum to a fibrotic tissue (5). Similar microstructural alterations of the erectile tissue had already been detected by other authors in patients with severe arterial insufficiency (6).

This phenomenon may affect not only the quality of erection of the operated patients but also the next options of treatment of the erectile deficiency.

The administration of Sildenafil soon after radical prostatectomy appears to exert an antifibrotic effect in the corpora cavernosa of prostate cancer patients with a consequent recovery of erectile function (7).

The fibrotic process following a radical prostatectomy (5) could be the cause of a chang- ing in the corpora cavernosa structure, with their retraction and a consequent shortening in penile lenght.

The authors concluded that the well-timed assumption of tadalafil in patient underwent radical prostatectomy, could inhibit, as it has been shown with the post-operative administration of sildenafil (7), the fibrotic process, avoiding the corpora cavernosa retraction, the conseguent penile shortening leading to a significant improvement in the recovery of erectile function.

Lately, the use of a new natural compound (Tradamix $\left.{ }^{\circledR}\right)$ containing potent antioxidant (8-10) is giving encouraging good result (data not published) on the prevention of the corpora cavernosa fibrosis in patients that underwent RP and in the treatment of Peyronie's disease.

In the treatment of mild, moderate ED and of the degenerative, dysfunctional process of the corpora cavernosa, the assumption of natural compound shows clear advantages compared to the several side effects and the many pharmacological incompatibilities observed with the use of 5PDE inhibitors (11). 


\section{REFERENCES}

1. Goldstein AM, Padma-Nathan H: The microarchitecture of the intracavernosal smooth muscle and the cavernosal fibrous skeleton. J Urol. 1990; 144: 11446.

2. Goldstein AM, Meehan JP, Morrow JW, Buckley PA, Rogers FA: The fibrous skeleton of the corpora cavernosa and its probable function in the mechanism of erection. Br J Urol. 1985; 57: 574-8.

3. Iacono F, Barra S, de Rosa G, Boscaino A, Lotti T: Microstructural disorders of tunica albuginea in patients affected by impotence. Eur Urol. 1994; 26: 2339.

4. Nehra A, Azadzoi KM, Moreland RB, Pabby A, Siroky MB, Krane RJ, et al.: Cavernosal expandability is an erectile tissue mechanical property which predicts trabecular histology in an animal model of vasculogenic erectile dysfunction. J Urol. 1998; 159: 2229-36.

5. Iacono F, Giannella R, Somma P, Manno G, Fusco F, Mirone V: Histological alterations in cavernous tissue after radical prostatectomy. J Urol. 2005; 173: 16736.

6. Persson C, Diederichs W, Lue T, Yen TSB, Fishman I J, McLin PH, et al.: Correlation of alterated penile ultrastructure with clinical arterial evaluation. J. Urol. 1989; 142: 1462-68.
7. Iacono F, Prezioso D, Somma P, Chierchia S, Galasso R, Micheli P: Histopathologically proven prevention of post-prostatectomy cavernosal fibrosis with sildenafil. Urol Int. 2008; 80: 249-52.

8. Iacono F, Prezioso D, Iapicca G, Taglialatela D, Ruffo A, Miclavez A: Saponins, Phlorotannins and Glycosamine contained in Tribulus Terrestris, Biovis and Alga Ecklonia Cava in the treatment of Erectile Dysfunction : A Preliminary Study. Eur.Journ. Sexuology and Sexual Health. 2010; 19(Suppl 1).

9. Iacono F, Prezioso D, Iapicca G, Ruffo A, Romis L, Di Lauro G, et al.: Evaluating the efficacy improving male sexual function with a new natural compound made of tribulus terrestris, Biovis and alga ecklonia cava and its synergic anti-aging action. J.M.H. 2010; 7: 305 .

10. Iacono F, Prezioso D, Ruffo A, Romis L, Di Lauro G: Treating erectile dysfunction with a new natural compound made of Alga Ecklonia, Tribulus Terrestris and Biovis and its antioxidant, antifibrotic and antiaging action. J Sex Med. 2010; 7(suppl 6): 431.

11. Tsertsvadze A, Yazdi F, Fink HA, MacDonald R, Wilt TJ, Bella AJ, et al.: Oral sildenafil citrate (viagra) for erectile dysfunction: a systematic review and metaanalysis of harms. Urology. 2009; 74: 831-836.e8.

Dr. Fabrizio Iacono Associate professor of Urology University "Federico II". Naples. Italy

Dr. Antonio Ruffo Resident in Urology University "Federico II". Naples. Italy. E-mail: antonio.ruffo7@gmail.com 